\title{
Inferior Vena Cava Filter Migration to the Right Ventricle: A Case Report and Review of Filter Migration and Misdeployment
}

\author{
Jason Dreyer ${ }^{\mathrm{a}, \mathrm{d}}$, Ketul Patel ${ }^{\mathrm{b}}$, Huma Shujaat ${ }^{\mathrm{b}}$, Michael Lee ${ }^{\mathrm{c}}$
}

\begin{abstract}
Inferior vena cava (IVC) filters are often seen as a safe and effective means of preventing pulmonary embolus in patients with contraindication to standard medical therapy. We present a case of a 57-year-old African-American man who presented with an IVC filter in his right ventricle. At our institution, percutaneous retrieval is initially attempted to reduce perioperitive morbidity.
\end{abstract}

Keywords: Inferior vena cava; IVC; Filter; Migration; Misdeployment; Complication

\section{Introduction}

Venous thromboembolism (VTE) is a significant cause of morbidity and mortality worldwide. The incidence of VTE is approximately 100 individuals per 100,000 each year. Deep venous thrombosis (DVT) accounts for approximately $66 \%$ of all VTE [1]. The vast majority of VTE are effectively managed with medical therapy such as systemic anticoagulation. Thrombi formation in the lower extremities or pelvis can embolize proximally to the pulmonary arteries. For this reason, inferior vena cava (IVC) filters are often utilized as a safe and effective means of preventing pulmonary embo-

\footnotetext{
Manuscript accepted for publication June 22, 2011

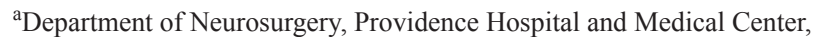
Southfield, MI, USA

${ }^{\mathrm{b}}$ Department of Internal Medicine, Providence Hospital and Medical Center, Southfield, MI, USA

${ }^{c}$ Department of Cardiothoracic Surgery, Providence Hospital and

Medical Center, Southfield, MI, USA

${ }^{\mathrm{d}}$ Corresponding author: Dr. Jason Dreyer, Providence Hospital, 16001

W 9 Mile Rd. Southfield, MI 48076, USA.

Email: greymew@yahoo.com
}

doi:10.4021/jmc240w lism (PE). Interruption of the inferior vena cava has been employed in the form of IVC filters in a subset of patients in whom anticoagulation is not suitable. The subset would include persons who are at high risk for developing PE, who are unresponsive to anticoagulation therapy, or in whom anticoagulation is contraindicated (i.e. recent neurosurgery, craniospinal trauma, hemorrhagic stroke, gastrointestinal bleeding, recurrent DVT or PE despite anticoagulation).

In the largest randomized study of patients with symptomatic DVTs, IVC filter placement along with anticoagulation reduced incidence of developing PEs versus anticoagulation alone ( $1 \%$ and $5 \%$ respectively). This benefit was seen during the first 12 days after randomization; however no statistical difference was seen in patients at two years with respect to survival or symptomatic PE. At eight years, overall mortality was similar in both groups [2].

There are a variety of IVC filters available, each with its own characteristics, advantages, and disadvantages, which have been proven effective at preventing PE. Currently there are scarce reports of adverse events related to the Cordis TrapEase filter in the form of migration [3-5]. At our institution the TrapEase filter is favored because of its relatively simple placement, low profile introducing system, and low rate of complications.

We describe a case of a man who presented 8 days after placement of a Cordis TrapEase IVC filter, which migrated to the right ventricle and was subsequently surgically removed.

\section{Case Report}

A 57-year-old African-American man with mild mental retardation, epilepsy, hypertension, diabetes mellitus, and chronic kidney disease, presented to the emergency department with complaints of sudden-onset shortness of breath, chest pain and "heaviness," associated with profuse sweating. Further workup was performed and the patient was diagnosed with acute DVT of the left lower extremity, and a pulmonary embolus of the right upper lobe.

An extensive discussion regarding the risks and benefits of anticoagulation was performed. Due to his seizure disorder and recent history of falls of unidentified cause (5-6 in 


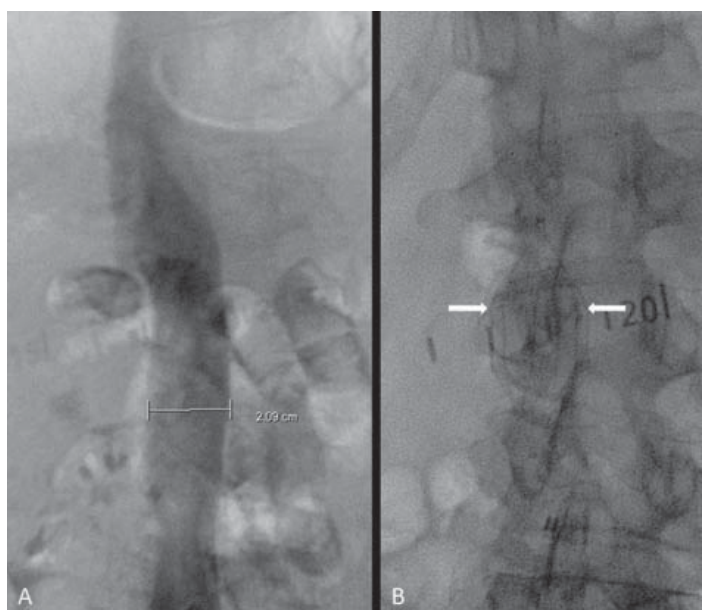

Figure 1. (A) Inferior vena cavogram showing a diameter of approximately $21 \mathrm{~mm}$. (B) Deployed TrapEase filter (between arrows).

the last month), the decision was made to place an IVC filter.

The patent was initially placed on a heparin drip and interventional radiology was consulted. A venacavogram was performed and he was found to have a normal caliber IVC (Fig. 1A). A Cordis TrapEase IVC filter was deployed at the origin of the right renal vein without complication (Fig. 1B). The patient tolerated the procedure well, and he was discharged the following day.

The patient was brought to the Emergency Department eight days later by his mother. She stated that "he is sleeping all day," which was uncharacteristic for him.

Chest radiography revealed a foreign body consistent with an IVC filter in the vicinity of the tricuspid valve (Fig. 2). Echocardiography demonstrated an echo-refractile structure within the right ventricle (RV) with multiple echo-densities within the right atrium (RA), RV, and right ventricular outflow tract (RVOT) (Fig. 3). The laboratory values were significant for a platelet count of $34,000 / \mu \mathrm{L}$. The patient was

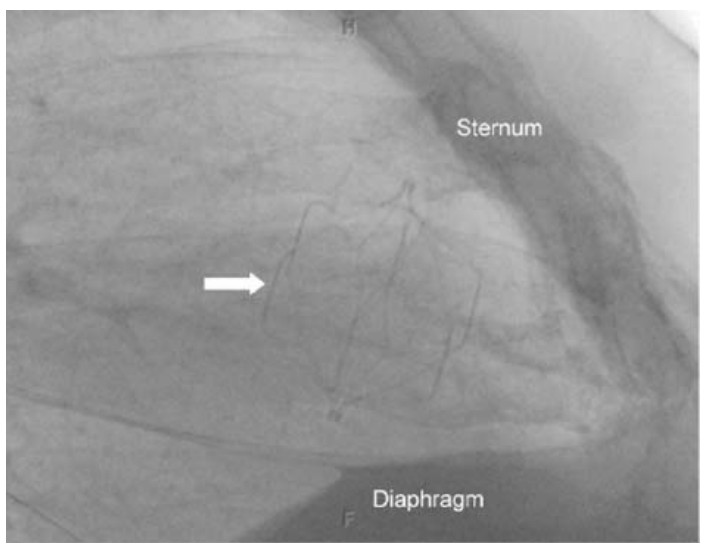

Figure 2. Lateral chest radiograph showing the location of the IVC filter in the heart (arrow).

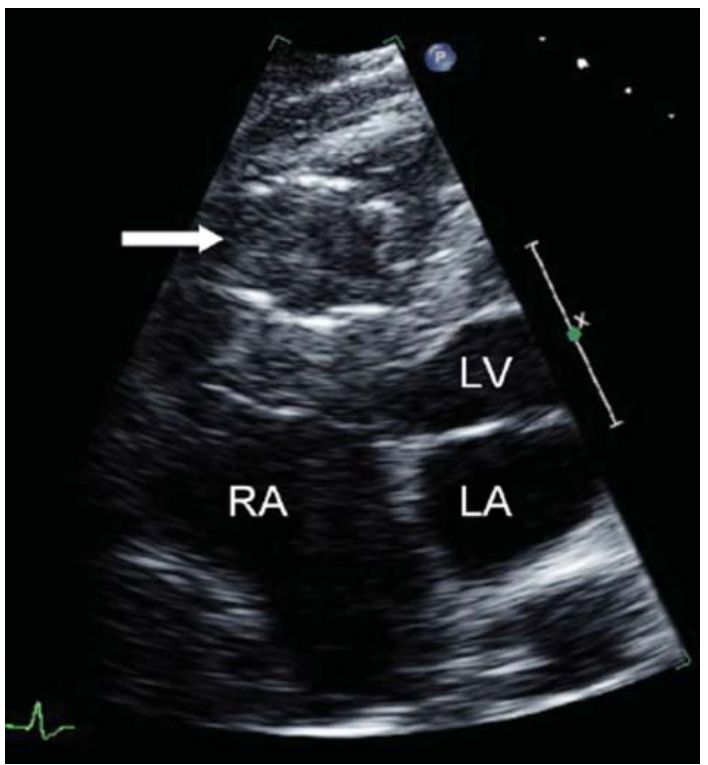

Figure 3. Apical, four-chamber, echocardiogram showing the presence of an IVC filter in right ventricle (arrow). The right atrium (RA), left ventricle (LV), and left atrium (LA) are labeled.

anticoagulated with argatroban, a direct thrombin inhibitor, pending the results of a heparin-induced thrombocytopenia (HIT) panel. Thrombotic thrombocytopenic purpura (TTP), idiopathic thrombocytopenic purpura (ITP), and HIT were considered and ruled out following a negative peripheral smear and the HIT panel.

Interventional radiology determined that percutaneous retrieval of the IVC filter was too high risk due to the involvement of the chordae tendineae. Therefore, the patient was admitted to the cardiovascular intensive care unit for

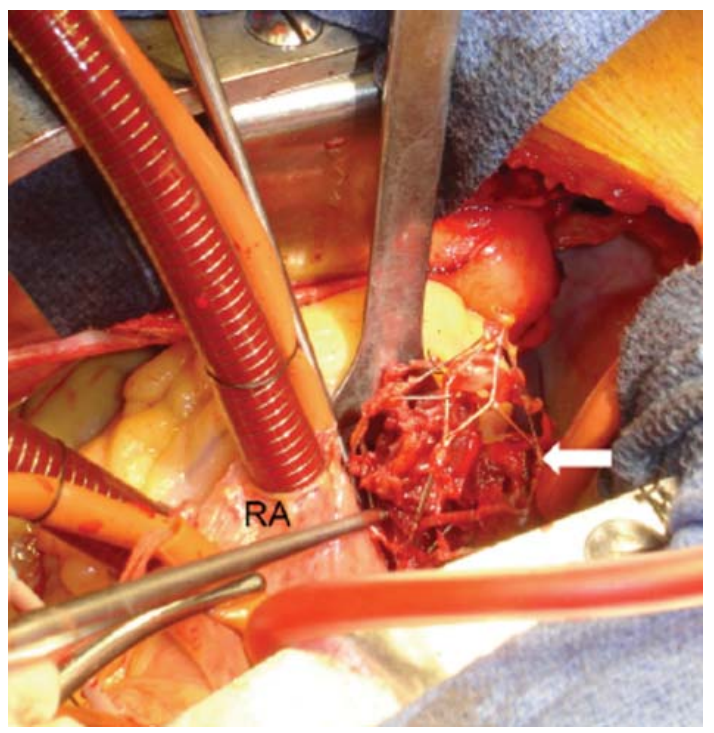

Figure 4. Excision of the IVC filter from the right ventricle (arrow). The right atrium (RA) is labeled. 


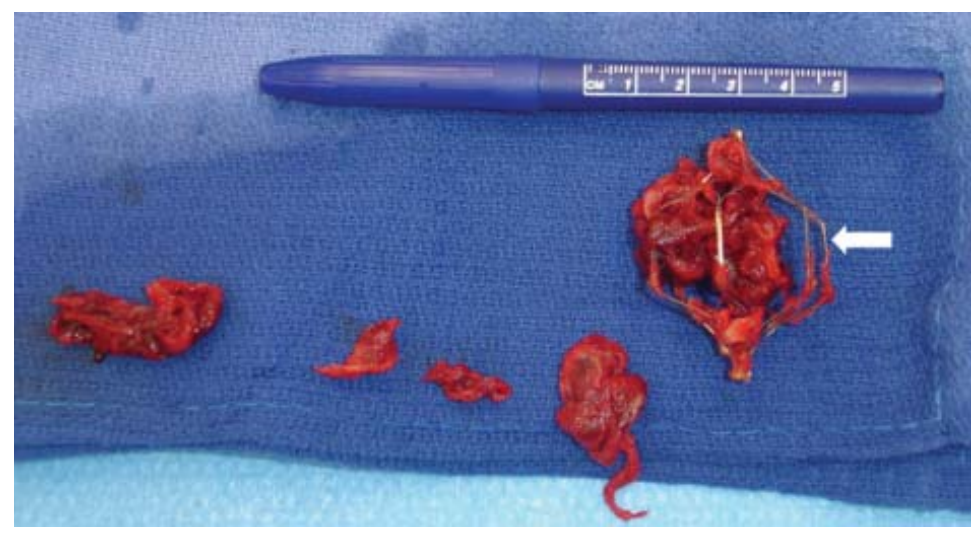

Figure 5. Excised IVC filter (arrow) with multiple cords of thrombi.

urgent cardiothoracic evaluation and treatment.

The patient underwent exploratory cardiac surgery. Utilizing cardio-pulmonary bypass, an excision of the $3.5 \times 3.5$ $\times 5.0 \mathrm{~cm}$ Cordis TrapEase IVC filter from the RV was performed. Multiple cords of thrombi were removed, and the damaged tricuspid valve was repaired (Fig. 4, 5).

The patient's post operative course was complicated with neurological deterioration requiring further investigations. Magnetic resonance imaging (MRI) of the brain demonstrated findings most consistent with bilateral watershed zones of infarction, likely related to a hypotensive/low flow state. Electroencephalogram demonstrated a diffusely encephalopathic state. Due to the patient's prior comorbidities and multiple organ failure, the decision was made to place the patient under the care of hospice on hospital day fourteen. At the request of the family, he was taken to home hospice, and died three days later.

\section{Discussion}

IVC filter placement to achieve partial mechanical interruption of the inferior vena cava is a well established method of preventing life-threatening PE caused by DVT [6]. In 1865, Armand Trousseau proposed the concept of creating a physical barrier to prevent emboli. Over the years many advances have been made to the concept of vena caval interruption. It initially began as surgical ligation of the femoral veins in the 1930s, and eventually led to the development of the MobinUddin filter in 1970. The stainless-steel Kimray-Greenfield filter, introduced in 1973, revolutionized the field of vena caval interruption. In the 1980 s further advancements made it possible for percutaneous placement of the IVC filters, and more recently, the development of retrievable filters [1].

The many styles of IVC filters on the market can be broken into three general categories: permanent, optional, and temporary. Permanent filters are intended to be left indefinitely within the IVC. Optional filters may be left indefinitely, but have a retrieval system if necessary. Temporary filters are intended to be removed after a short period of time, and remain attached to the patient's skin by an intraluminal fixation system.

Each IVC filter type and style has its own advantages and disadvantages. Characteristics such as available access site, profile of introducer, patency of the filter to blood flow, retrievability, and interventionalist's preference determine which filter is placed.

The permanent filters currently on the market in the United States include: Greenfield stainless steel and titanium (Boston Scientific/Meditech), Bird's Nest (Cook), Simon Nitinol (Bard), VenaTech LP and LGM (B. Braun), and TrapEase (Cordis). The optional filters include: Gunther Tulip (Cook), OptEase (Cordis), and G2 (Bard). The only temporary filter available is the Tempo Filter II (B. Braun). There are many other filters not mentioned here available outside the United States, in clinical trials, or in premarketing.

The TrapEase filter is a double-basket, symmetrical device made from a single nitinol tube. The device has a nonexpanded maximum length of $65 \mathrm{~mm}$, which reduces to 50 $\mathrm{mm}$ when expanded to its maximum diameter of $35 \mathrm{~mm}$; six straight struts connect the proximal and distal baskets. The proximal and distal basket is composed of six petal-shaped openings, which are connected by six straight struts. A proximal and distal hook is located on each connecting strut for fixation of the filter to the vena cava wall [5].

Complications of IVC filters can occur during placement, immediately following the procedure, or months to years later [7]. Hann et al. describes procedure-related complications including misplacement (1.3\% of insertions), pneumothorax $(0.02 \%)$, hematoma $(0.6 \%)$, air embolism $(0.02 \%)$, carotid artery puncture $(0.04 \%)$, and arteriovenous fistula $(0.02 \%)$. Fatalities caused by these complications are rare; they occur in only $0.13 \%$ of insertions. A common early post-procedure complication of filter placement is insertion site thrombosis (0.4-36\%). Delayed complications of filter placement include: IVC thrombosis (2-10\%), filter migration $(0.3 \%)$ [8], IVC penetration $(0.3 \%)$ [9], and filter disruption from entrapment of guide wires [1]. 
Migration is a rare complication of IVC filter placement. It is defined as shifting of the IVC filter more than $1 \mathrm{~cm}$ in a cranial or caudal direction [9]. Migration of the filter to the heart or pulmonary arteries is uncommon.

Signs and symptoms of IVC filter migration to the heart include: chest pain, hypotension, dyspnea, tachycardia, premature ventricular contractions, right bundle branch block, asystole, neck pain, atrial fibrillation, confusion, lightheadedness, diaphoresis, and nausea. The patient in this case presented with symptoms of confusion and lethargy for approximately one week. Two cases have been reported with asymptomatic patients. In those cases, one filter was located in the right atrium and the other was in the right pulmonary artery $[10,11]$.

Causes of filter migration can be grouped into three categories: mechanical, iatrogenic, and physiologic. Mechanical causes include failure of the device delivery system or the filter itself. These occurrences are usually realized during the implantation procedure and should be reported to the FDA and the device manufacturer.

Iatrogenic causes of filter migration include entanglement with guide wires for central venous line placement and attempting to place a filter without performing an inferior vena cavogram to properly size the device. Each filter is approved for deployment up to a specific inferior vena cava diameter. For example, the TrapEase filter is approved for inferior vena cava diameters $18-30 \mathrm{~mm}$ [5].

Physiologic causes of migration may result from temporary dysmorphism of the inferior vena cava. Bending, coughing, or Valsalva maneuvers may allow dislodgement of the filter. A "sail effect" in which blood flow might induce cephalad migration of conical filters was proposed by Rossi et al [12]. The force of a large thrombus striking the filter may be enough to cause dislodgement. Finally, a filter which already is bearing a burden of clot in addition to an insult listed above may synergistically induce migration.

The TrapEase IVC filter has been the subject of four clinical studies, two prospective, and two retrospective from 2001 to 2006 . Among the 1,047 subjects, there were no cases of filter migration [5, 13-15]. Only two previous case reports of TrapEase filter migration to the heart have been published in the literature. In one case the patient died of a sudden cardiac arrest three days after filter deployment. The filter was found within the tricuspid valve on autopsy [3]. In the second case, the patient presented with dyspnea, hypotension, and confusion seven days following the filter placement. The filter was surgically removed and the patient was discharged on oral anticoagulation [4].

\section{Conclusion}

The number of IVC filters placed has increased from 2,000 in 1979 to 49,000 in 1999 [16]. In the literature, there are two reported cases of the TrapEase filter migration to the heart or pulmonary outflow tract. Our case is only the third occurrence; therefore, TrapEase IVC filter migration to the heart after successful placement is an extremely rare complication.

From our literature review of migrated and misdeployed filters, initial percutaneous intervention was successful in twenty-four out of thirty-one $(77 \%)$ cases. When the filter could not be retrieved percutaneously, surgical excision was successful in three out of four $(75 \%)$ cases, and the no further interventions group had four patients out of five (80\%) survive at six, thirty-six, sixty, and eighty months followup respectively. Primary surgical intervention has been successful in fourteen out of nineteen (74\%) cases since 1976, including our case. Six of the fifteen $(40 \%)$ patients that did not undergo any intervention survived nine to forty-eight months.

Based on the data collected, it is difficult to make treatment recommendations. Percutaneous and surgical interventions have similar survival. At our institution, percutaneous retrieval is preferred, when possible, to minimize peri-operative morbidity. If the patient is too high risk for surgery and percutaneous intervention is not possible or unsuccessful, leaving the filter in place may be considered. In that situation, anticoagulation and/or a second filter are advisable.

\section{Conflict of Interest}

There is no grant support or conflict of interest to report by any of the authors.

\section{References}

1. Hann CL, Streiff MB. The role of vena caval filters in the management of venous thromboembolism. Blood Rev. 2005;19(4):179-202.

2. Decousus H, Leizorovicz A, Parent F, Page Y, Tardy B, Girard P, Laporte S, et al. A clinical trial of vena caval filters in the prevention of pulmonary embolism in patients with proximal deep-vein thrombosis. Prevention du Risque d'Embolie Pulmonaire par Interruption Cave Study Group. N Engl J Med. 1998;338(7):409-415.

3. Haddadian B, Shaikh F, Djelmami-Hani M, Shalev Y. Sudden cardiac death caused by migration of a TrapEase inferior vena cava filter: case report and review of the literature. Clin Cardiol. 2008;31(2):84-87.

4. Porcellini M, Stassano P, Musumeci A, Bracale G. Intracardiac migration of nitinol TrapEase vena cava filter and paradoxical embolism. Eur J Cardiothorac Surg. 2002;22(3):460-461.

5. Rousseau H, Perreault P, Otal P, Stockx L, Golzarian J, Oliva V, Reynaud P, et al. The 6-F nitinol TrapEase 
inferior vena cava filter: results of a prospective multicenter trial. J Vasc Interv Radiol. 2001;12(3):299304.

6. Goldfaber SZ, Grassi CJ. Management of pulmonary embolism. In: Sabeston DC, ed. Textbook of surgery, 8th ed. Philadelphia: WB Saunders, 1990;115-127.

7. Ray CE, Jr., Kaufman JA. Complications of inferior vena cava filters. Abdom Imaging. 1996;21(4):368-374.

8. Streiff MB. Vena caval filters: a review for intensive care specialists. J Intensive Care Med. 2003;18(2):5979 .

9. Mitchell WB, Bonn J. Percutaneous retrieval of a Greenfield filter after migration to the left pulmonary artery. J Vasc Interv Radiol. 2005;16(7):1013-1017.

10. Patel A, Cholankeril J. Case of the migrating embolic filter. Hosp Pract. 1991;26(4):129-132.

11. Rao KM, Simons AJ, Hare CL, Smulyan H. Migration of a Kimray-Greenfield filter into the pulmonary artery: localization by transesophageal echocardiography. Am Heart J. 1993;125(2 Pt 1):543-544.

12. Rossi P, Arata FM, Bonaiuti P, Pedicini V. Fatal outcome in atrial migration of the Tempofilter. Cardiovasc Intervent Radiol. 1999;22(3):227-231.

13. Schutzer R, Ascher E, Hingorani A, Jacob T, Kallakuri S. Preliminary results of the new $6 \mathrm{~F}$ TrapEase inferior vena cava filter. Ann Vasc Surg. 2003;17(1):103-106.

14. Kalva SP, Wicky S, Waltman AC, Athanasoulis CA. TrapEase vena cava filter: experience in 751 patients. $\mathrm{J}$ Endovasc Ther. 2006;13(3):365-372.

15. Liu WC, Do YS, Choo SW, Kim DI, Kim YW, Kim DK, Shin SW, et al. The mid-term efficacy and safety of a permanent nitinol IVC filter(TrapEase). Korean J Radiol. 2005;6(2):110-116.

16. Stein PD, Kayali F, Olson RE. Twenty-one-year trends in the use of inferior vena cava filters. Arch Intern Med. 2004;164(14):1541-1545. 